\title{
APLICAÇÃO DE TÉCNICAS DE INTELIGÊNCIA ARTIFICIAL EM CONTROLE DE PROCESSOS EM BATELADA
}

\author{
Mayer Fernandes dos Santos SIlva1; Herman Augusto Lepikson² \\ 1 Avenida Orlando Gomes, Piatã, 1845 (41650-010); Salvador/Bahia; \\ mayer_eq@hotmail.com \\ ${ }^{2}$ Avenida Orlando Gomes, Piatã, 1845 (41650-010); Salvador/Bahia
}

\begin{abstract}
Resumo: O objetivo deste trabalho é realizar uma revisão da literatura sobre o uso de inteligência artificial (IA) no processo em batelada, caracterizando o seu benefício no tratamento da não linearidade e transiência de variáveis de processos em batelada. Através de uma revisão sistemática da literatura, foram estabelecidos critérios para busca e seleção dos artigos, de forma a retratar a evolução de estudos na área nos últimos vinte anos. Após seleção, as principais aplicações de IA para controle de bateladas foram descritos. Foi possível concluir que a base Science Direct apresentou o maior número de artigos na área e que de modo geral variações de processo foram tratadas através de redes neurais artificiais e lógicas fuzzy demonstraram capacidade de auxiliar no controle de variáveis não lineares.
\end{abstract}

Palavras-Chave: Controle de Processo; Inteligência Artificial; Processo em Batelada; Redes Neurais; Lógica Fuzzy.

\section{APPLICATION OF ARTIFICIAL INTELLIGENCE TECHNIQUES IN BATCH PROCESS CONTROL}

\begin{abstract}
The objective of this paper is to review the Iterature on the use of artificial intelligence $(\mathrm{Al})$ applied to batch process control, characterizing its benefit in the treatment of nonlinearity and transience of batch process variables. Through a systematic review of the literature, criteria were established for searching and selecting articles, in order to analyze the evolution of studies in the area in the last twenty years. After selection, the main applications of Al for batch control were described. It was possible to conclude that the Science Direct database presented the largest number of articles in the area and that in general process variations were treated through artificial neural networks and fuzzy logic demonstrates the ability to address nonlinearities of control variables.
\end{abstract}

Keywords: Process Control; Artificial Intelligence; Batch Process; Neural Networks; Fuzzy Control. 


\section{INTRODUÇÃO}

Empresas de manufatura devem estar aptas a reagir em determinadas situações de mercado e manter a eficiência de seus processos em diferentes faixas de operação de modo à se manter bem posicionada na crescente competitividade e dinâmica do mercado atual. Tecnologias baseadas em análises big data, aprendizado de máquina e métodos de inteligência artificial (IA) são implementadas com o objetivo de monitorar e otimizar processos. Estas técnicas preditivas aplicadas a processos de manufatura podem, além de otimizar processos, embasar decisões de negócios através de dados em tempo real com maior agilidade e eficiência [1].

Neste cenário, processos em batelada tem se tornado cada vez mais importantes devido à crescente competição econômica global e natureza específica de processos industriais que não possuem competitividade no modo contínuo de produção. Unidades em batelada são mais flexíveis, pois permitem a implementação de equipamentos com multiobjetivos, permitindo produção de compostos diferentes em uma mesma linha. Tendo em vista que processos em batelada possuem comportamentos não lineares, estado de transiência e variáveis discretas que elevam o desafio do controle do processo, há um estímulo ao estudo da utilização de controles preditivos baseados em modelos empíricos para melhor controle do processo [2]. Para lidar com não linearidades e tratar variações em condições operacionais que afetam o controle de processo é necessário desenvolver controles inteligentes e preditivos que possibilitem identificar de forma antecipada o comportamento do processo frente a distúrbios operacionais [3].

Controles do tipo feedback atuam basicamente no erro da variável controlada e não possuem capacidade de identificar modelos e controlar processo baseados em seu comportamento teórico. Esta estratégia de controle possui limitações quando aplicada a processos não lineares e discretos, inviabilizando sua aplicação em determinados controles de processos em batelada. Controles feedforward, por sua vez, possuem capacidade de trabalhar sob modelo previamente especificado e, em alguns casos mais avançados, possuem poder de se ajustarem ao processo de acordo com variações através de técnicas de aprendizagem de máquina [4].

\section{METODOLOGIA}

Este trabalho teve como método a revisão de literatura da área de modo a identificar artigos científicos sobre o tema de inteligência artificial e aplicabilidade no controle de processos industriais, especificamente nos processos em batelada. Para isso, foi definido um conjunto de palavras chave e período para seleção do material a ser trabalhado, de forma a encontrar artigos que abordem o tema, indicando tendências de linha de pesquisas e sua evolução. 


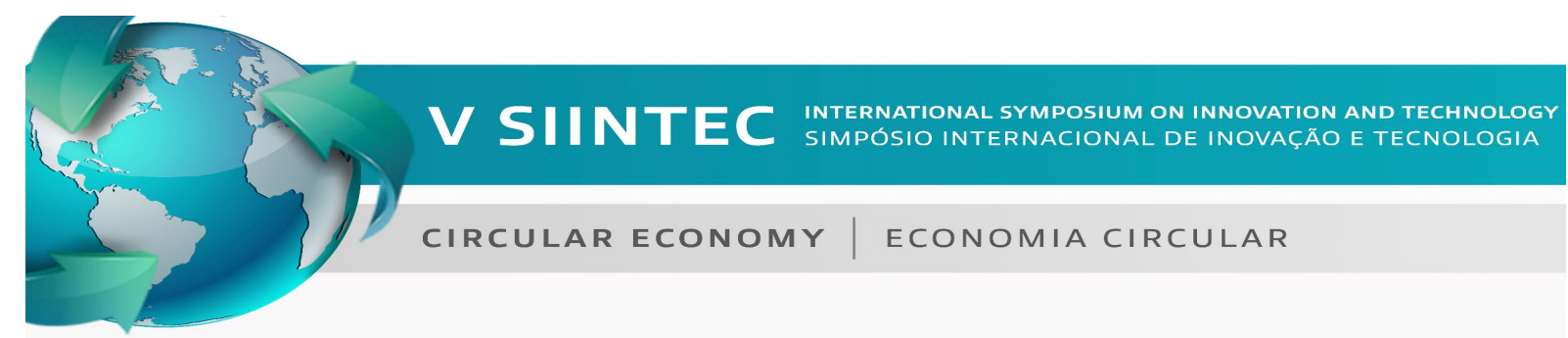

A base de pesquisa utilizada foi o google acadêmico, onde, para alcançar um resultado mais acurado quanto ao assunto pesquisado, foram definidas as seguintes strings:

("Artificial Intelligence" OR "Machine Learning") AND ("Process Control" OR "Batch Control") AND ("Industrial" OR "Industry")

O período de pesquisa foi definido como os últimos vinte anos (1999 a 2019) de forma a encontrar artigos desde mais antigos, que indicam trabalhos em época onde os computadores não possuíam grande capacidade de processamento, até os dias de hoje onde a capacidade computacional cresce de forma exponencial. Além disto, pode-se verificar a tendência de estudo da área ao longo dos anos.

Foram também definidos critérios para seleção dos artigos encontrados de modo a refinar a os resultados obtidos, incluindo na análise o material com maior aprofundamento no assunto abordado. Para inclusão ou exclusão de artigos na pesquisa foram utilizados os seguintes critérios:

- Aprofundamento do motivo para se utilizar técnicas de inteligência artificial na resolução de problemas em unidades industriais;

- Aplicação de técnicas de inteligência artificial para controle de processos contínuos e em bateladas;

- Utilização de inteligência artificial na predição de comportamento de processos industriais.

Figura 1. Fluxo de Seleção dos Artigos
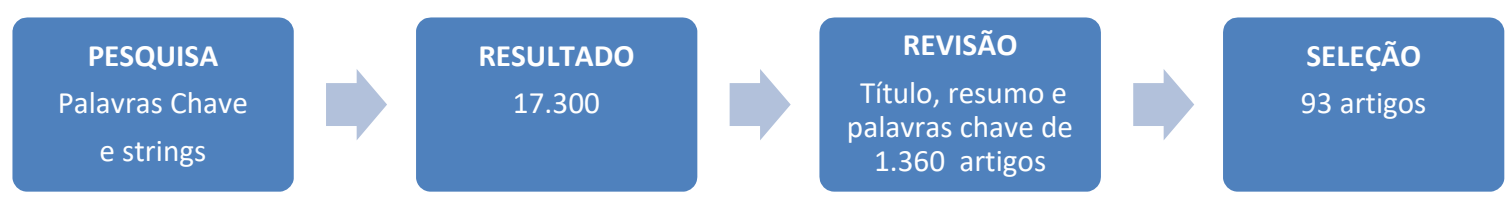

Após a seleção dos artigos de maior aderência aos critérios estabelecidos, foi abordado de forma geral os tipos de algoritmos e estratégias mais utilizados para otimização de processos industriais. Após esta abordagem, buscou-se entender como as características e desafios de processos em batelada podem ser tratados através do uso de técnicas de aprendizado de máquina e IA, de forma a auxiliar na melhoria de performance em controle de processos.

\section{RESULTADOS E DISCUSSÃO}

Através da busca realizada, foi possível observar que a base com maior número de artigos publicados na área foi a Science Direct. Outras bases como IEEExplore Springer Link e Research Gate também apresentaram artigos relevantes, porém em menor número. Outros artigos de anais, revistas e teses compuseram o restante do material encontrado. A tabela abaixo demonstra o número de artigos encontrados na área por base de dados científicos. 
Tabela 1. Título da tabela

\begin{tabular}{|l|c|}
\hline Bases de Dados & Artigos Encontrados \\
\hline Science Direct & 41 \\
\hline IEEExplore & 7 \\
\hline Springer Link & 5 \\
\hline DResearch Gate & 2 \\
\hline Outros & 38 \\
\hline
\end{tabular}

A estratificação dos artigos encontrados por data indica que há um número crescente de publicações na área de aprendizado de máquina aplicada a controle de processos. No entanto, artigos importantes em base teórica e primeiras aplicações também foram publicados no final da década de 1990 e início dos anos 2000 servindo de base para os estudos mais recentes. A figura 2 ilustra os resultados obtidos por ano.

Figura 2. Artigos Selecionados

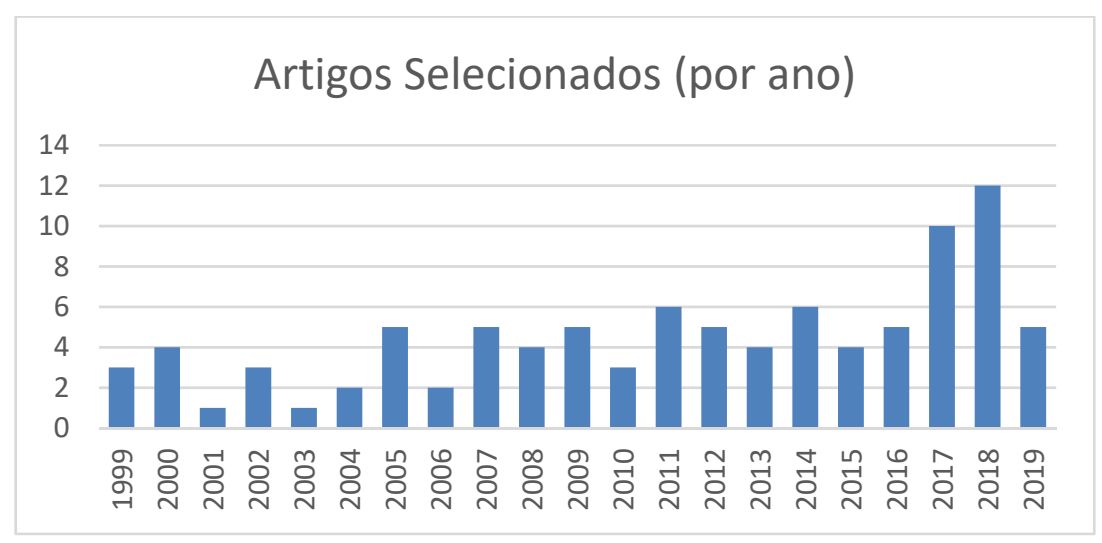

Foi possível perceber que os artigos mais recentes, além de maior número, possuem maior diversidade de técnicas como tipos de algoritmos e estratégias baseadas em inteligência artificial e aprendizado de máquina. Estes Estudos têm provado que algoritmos de aprendizagem e otimização são eficazes e possuem potencial de elevar eficiência de equipamentos industriais, sobretudo na indústria por batelada. A ideia básica desta abordagem inclui a modelagem do processo, estimando valores futuros e uso de predições para otimização. 


\subsection{Algumas Técnicas de IA aplicadas ao Controle e Monitoramento de Processos e Equipamentos Industriais}

Redes Neurais Artificiais (RNA) é uma das técnicas de inteligência artificial com grande aplicabilidade na indústria química, utilizando conceito aprendizado de forma similar ao que acontece com o cérebro humano. No corpo humano a rede de neurônios possui importante papel na realização de tarefas do dia-a-dia. São milhões e milhões de neurônios conectados entre si processando informações em paralelo durante a realização de atividades. Esta rede biológica recebe e envia informações em tempo real permitindo a realização de tarefas complexas e aprendizado contínuo. Como ilustra a Figura 3, o neurônio artificial simula o comportamento do neurônio biológico, possuindo diversas entradas e uma saída [5]. A figura abaixo ilustra o modo de funcionamento de um neurônio artificial e o seu funcionamento de forma integrada, formando uma rede com camadas definidas:

Figura 3. Neurônio Artificial e Rede Neural Artificial Multicamadas [2]
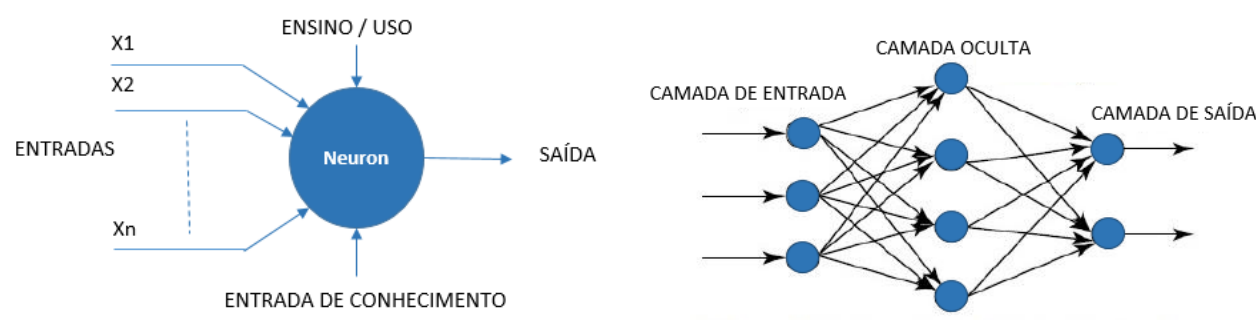

Em Seu estudo, [6] relatou que algumas técnicas de IA que foram utilizadas em diferentes trabalhos para reduzir emissões de NOx em caldeiras industriais: genetic algorithms (GA), particle swarm optimization (PSO) e ant colony optimization (ACO). Além desta abordagem, [A] aplicou em seu estudo técnicas de Support Vector Regression (SVR), Artificial Neural Network (ANN), Extreme Learning Machine (ELM) e Harmony Search (HS) onde demonstrou os benefícios na aplicação de monitoramento deste tipo de equipamento de equipamentos de combustão.

Outra técnica presente em grande número de pesquisas a partir dos anos 2000 é o algoritmo de Extremum seeking, com sucesso de aplicação em vários equipamentos industriais. Esta técnica possui duas diferentes abordagens: (i) baseada em dados do processo ou baseada em dados de processo e conhecimento do processo de forma combinada. A primeira abordagem baseia-se em perturbações onde a função de otimização pode ser estimada e usada para otimizar custo ou performance. A segunda abordagem assume que a função de otimização é conhecida e possui parâmetros que podem ser dinamicamente ajustados [7].

Nas sessões a seguir, são descritas características de processos em batelada encontrados na literatura e como a utilização de técnicas de inteligência artificial podem ser utilizadas para monitoramento e controle preditivo. 


\subsection{Controle de Variáveis Não Lineares}

Para tratar a não linearidade do processo de neutralização do $\mathrm{pH}$, [3] elaborou lógica de controle fuzzy utilizando seis regiões de dinâmicas diferentes, com range entre 6 e 9. Esta lógica foi utilizada em combinação com estrutura feedforward inserindo redes neurais. Aplicou-se ainda técnicas de Genetic Algorithm (GA), Differential Evolution (DE) e Particle Swarm optimization (PSO) para otimização do controle de $\mathrm{pH}$, obtendo resultados satisfatórios, porém similares, concluindo que $\mathrm{DE}$ possui vantagem em relação a GA e PSO devido à maior simplicidade de aplicação.

Para controle ótimo de processos em batelada multiobjetivo, com variáveis de característica não lineares [8] utilizou uma rede neuro-fuzzy com modelo de predição. Foi desenvolvido um conjunto fuzzy com diferentes modelos dinâmicos "locais". Dados operacionais foram utilizados para treinamento da rede, identificando as funções locais, bem como os pesos correspondentes de cada variável e região. $\mathrm{O}$ modelo obtido apresentou capacidade adaptativa para diferentes objetivos de um reator em batelada.

\subsection{Variáveis discretas e Difícil Replicação de Condições em processos em Batelada}

Ao estudar um reator em batelada de polimerização de metacrilato, [9] abordou que o processo em batelada está susceptível a distúrbios e variações difíceis de serem previstos, devido à falta de correspondência à modelos teóricos, dificultando o controle do processo. No reator em estudo, foi implementado então um modelo de rede neural com predição de erros e realimentação de dados da batelada anterior. Foi possível minimizar os erros batelada a batelada, gradualmente, encontrando com sucesso um controle ótimo que reduz a variabilidade do processo.

Ao analisar um secador industrial em batelada [10] observou que há variações de parâmetros entre uma batelada e outra como tamanho, tempo de processamento e tempo de espera. Estas variações são umas das maiores preocupações na indústria por batelada. Através de complexo processo iterativo utilizando redes neurais para reconhecimento de padrões associado com controle fuzzy, foi possível reduzir tempo de análises utilizando decisões online, maximizando produtividade da unidade em estudo. Em seu estudo, abordou ainda que Computadores e hardware modernos com softwares inteligentes tornam possível o processamento de grande quantidade de dados com menor custo, possibilitando o uso de algoritmos mais complexos de inteligência artificial nos dias atuais. 


\subsection{Estados de Transiência em Bateladas}

Boa parte dos processos industriais envolvem bateladas que possui estados não estacionários, por natureza. A complexidade acrescentada ao controle de processos com variáveis não estacionárias foi retratada por [11], onde relata a necessidade de se abordar o estado de transiência de equipamentos como este em estudos científicos, abordando também desafios em transições de fases e outras variáveis dinâmicas e não lineares. Através da análise e uso do histórico de dados, foi possível criar sistema de monitoramento e controle do processo de fermentação possibilitando redução de variabilidade, aumento de produtividade e possibilitando monitoramento on-line.

[12] abordou como um biorreator batelada de produção de álcool apresenta variáveis com comportamentos não lineares e variações entre bateladas que demanda a necessidade de aplicação de modelos empíricos de aprendizado. Foi utilizada uma regressão generalizada utilizando redes neurais artificiais para monitoramento e modelagem do reator. Através deste modelo foi possível realizar acurada predição de saídas de processo, rápido treinamento da rede, se comparado à técnica de back-propagation do erro, possibilitando a predição valores de variáveis com complexo estado de transiência durante a batelada.

Em seu estudo para determinar a composição de um vaso intermediário em uma coluna de destilação não convencional, [13] relata que a taxa de carga do equipamento varia ao longo do tempo e composição ao longo do tempo que devem ser preditas durante toda a batelada para evitar que produtos fiquem fora de especificação, necessitando reprocessamento da batelada. Para criação de um sensor virtual de concentração da batelada foi construído uma rede neural artificial recorrente com três camadas, a qual foi treinada e testada, apresentando boa acuracidade.

\section{CONCLUSÃO}

Este artigo teve como objetivo realizar uma revisão da literatura sobre o uso de IA aplicada ao controle de processos em batelada. Foi caracterizado de forma preliminar e geral o benefício de técnicas de IA no tratamento da não linearidade e transiência de variáveis de processos em batelada.

Foi possível concluir que o tema IA aplicado ao controle de processos, mais especificamente em processos em batelada, continua presente e cresce em número de publicações ao longo do tempo. A quantidade e sofisticação de técnicas cresceu nos últimos 20 anos em função dos recentes avanços de tecnologia e hardware que a indústria de informática e computação industrial tem proporcionado.

A base científica Science Direct apresentou maior número de publicações na área. Foi possível concluir também, através dos artigos selecionados, que os principais desafios do controle de processo em batelada são: (i) não linearidade de 
variáveis, (ii) estado de transiência das variáveis e (iii) difícil replicação de condições presentes em condições futuras. Para o tratamento destes desafios a literatura sugere o uso de técnicas de aprendizado de máquina (como redes neurais), subdivisão das variáveis em regiões e representação via modelos (fuzzyficação) e técnicas numéricas de regressão online de modelos, respectivamente. Uma forma amplamente utilizada e testada para controle deste tipo de processo é a combinação de redes neurais com lógica fuzzy.

\section{Agradecimentos}

Agradeço a Deus por ter me dado saúde e força para superar as dificuldades. Ao SENAI CIMATEC pelo ambiente criativo e amigável que proporciona. Ao meu orientador Herman Lepikson pela orientação, apoio e confiança. Agradeço à minha esposa, Fernanda, pelo amor, apoio e compreensão durante o período de pesquisa e a meus pais e irmãos pelo incentivo, amor e apoio incondicional que sempre me auxiliou durante as conquistas em minha vida.

\section{REFERÊNCIAS}

${ }^{1}$ MEHDJYEV, Nijat. Et al. Time Series Classification Using Deep Learning for process Planning: A Case from the Process Industry. Procedia Computer Science. Chicago, Illnois, 2017.

2 ALIPOOR, M. ZEINALI, M. YAZDI, S. Fuzzy Temperature Control in a Batch Polymerization Reactor Using ANFIS Method. International Journal of Engineering and Technology. Sabzevar, Iran, 2009.

${ }^{3}$ SINGH, P. Et Al. Design and Implementation of Intelligent Control Schemes for a $\mathrm{pH}$ Neutralization Process. WSEAS TRANSACTIONS on SYSTEMS and CONTROL. Karnataka, India, 2018.

4 TOMA, Liliana. COMPARATIVE ANALYSIS OF PH CONTROL METHODS. ACTA TECHNICA NAPOCENSIS Electronics and Telecommunications. Cluj-Napoca, Romania, 2016.

5 COSENZA, B. GALLUZO, M.Nonlinear fuzzy control of a fed-batch reactor for penicillin production. Computers and Chemical Engineering 36. Palermo, Italy, 2012.

6 TAN, P. Et Al. Modeling and optimization of NOX emission in a coal-fired power plant using advanced machine learning methods. Energy Procedia. Wuhan, China, 2014.

7 SAFADY, Luis Felipe. Control of a neutralization reactor using extremum-seeking approaches. University of Campinas. Campinas, Brazil, 2018. 
8 ZHANG, J. Modelling and Multi-Objective Optimal Control of Batch Processes Using Recurrent Neuro-fuzzy Networks. International Journal of Automation and Computing 1. Newcastle, United Kingdom, 2006.

${ }^{9}$ XIONG, Z. ZHANG, J. A batch-to-batch iterative optimal control strategy based on recurrent neural network models. Journal Of Process Control. New castle, UK, 2005.

10 SIMON, L. HUNGERBUHLER, K. Industrial batch dryer data mining using intelligent pattern classifiers: Neural network, neuro-fuzzy and Takagi-Sugeno fuzzy models. Chemical Engineering Journal 157. Zurich, Switzerland, 2010.

11 ALFORD, J. S. Bioprocess control: Advances and challenges. Computers and Chemical Engineering. Indianapolis, IN US, 2006.

12 NANDI, S. ARTIFICIAL INTELLIGENCE BASED METHODOLOGIES FOR MODELING, OPTIMIZATION AND MONITORING OF CHEMICAL PROCESSES. National Chemical Laboratory. Pune, India, 2010.

13 ZAMPROGNA, E. BAROLO, M. SEBORG, D. COMPOSITION ESTIMATIONS IN A MIDDLE-VESSEL BATCH DISTILLATION COLUMN USING ARTIFICIAL NEURAL NETWORKS. Institution of Chemical Engineers. Santa Barbara, USA, 2001. 